\title{
The Influence of Biologic Factors on the Surgical Decision in Advanced Neuroblastoma
}

\author{
Wen-Ming Hsu, MD, PhD, ${ }^{1}$ Yung-Ming Jen, MD, ${ }^{2}$ Hsinyu Lee, PhD, ${ }^{3}$ \\ Min-Liang Kuo, PhD, ${ }^{4}$ Po-Nien Tsao, MD, PhD,${ }^{5}$ Chiung-Nien Chen, MD, PhD, ${ }^{1}$ \\ Dar-Ming Lai, MD, ${ }^{1}$ Ming-Tsan Lin, MD, DMSc, ${ }^{1}$ Hong-Shiee Lai, MD, PhD, ${ }^{1}$ \\ Wei-Jao Chen, MD, MPH, DMSc, ${ }^{1}$ and Fon-Jou Hsieh, MD ${ }^{6}$
}

\footnotetext{
${ }^{1}$ Department of Surgery, National Taiwan University Hospital and National Taiwan University College of Medicine, \#7 Chung-Shan South Road, Taipei 100, Taiwan

${ }^{2}$ Department of Pathology, National Taiwan University Hospital and National Taiwan University College of Medicine, \#7 Chung-Shan South Road, Taipei 100, Taiwan

${ }^{3}$ Department of Life Science and Institute of Zoology, National Taiwan University, \#1, Sec. 4, Roosevelt Road, Taipei 100, Taiwan

${ }^{4}$ Institute of Toxicology, National Taiwan University College of Medicine, \#1, Sec. 1, Jen-Ai Road, Taipei 100, Taiwan

${ }^{5}$ Department of Pediatrics, National Taiwan University Hospital and National Taiwan University College of Medicine, \#7 Chung-Shan South Road, Taipei 100, Taiwan

${ }^{6}$ Graduate Institute of Clinical Medicine, National Taiwan University College of Medicine, \#7 Chung-Shan South Road, Taipei 100, Taiwan
}

\begin{abstract}
Background: Molecular markers greatly affect the outcome of neuroblastoma. This study evaluated the influence of Trk-A and myelocytomatosis viral-related oncogene, neuroblastoma-derived (MYCN) on the role of surgery in advanced neuroblastoma.

Methods: Ten stage 3 and 35 stage 4 neuroblastoma patients were included. Tumor resection was classified into gross total resection (GTR) and incomplete resection. Patients were classified into three biological risk groups according to Trk-A expression and myelocytomatosis viral-related oncogene, neuroblastoma-derived (MYCN) status in tumor tissues studied by immunohistochemistry and fluorescence in situ hybridization, respectively: low risk (positive Trk-A and normal MYCN), intermediate risk (negative Trk-A and normal MYCN), and high risk (positive or negative Trk-A and MYCN amplification). The effect of tumor resection on prognosis was studied and stratified according to the risk grouping.

Results: GTR was achieved in 21 patients $(46.7 \%)$ with a higher complication rate (33\% vs. $8 \%$ in the incomplete resection group, $P=.036)$. GTR was easier to achieve in low-risk tumors than in intermediate- or high-risk tumors ( 12 of 13,4 of 17 , and 5 of 15 , respectively; $P$ $<.001)$. GTR predicted a favorable prognosis for intermediate-risk patients $(P=.037 ; \log$ rank test), but not for low- or high-risk patients because of the overall favorable and poor prognosis, respectively.

Conclusions: GTR carries a potentially higher possibility of complication. Although GTR can be achieved easily in low-risk neuroblastoma patients with a favorable prognosis, surgeons should do their best to achieve GTR for intermediate-risk patients to improve outcome. Nevertheless, sacrificing vital organs to achieve GTR for high-risk patients is not justified.
\end{abstract}

Key Words: Neuroblastoma-Surgery-Trk-A-MYCN.

Received October 15, 2004; accepted August 24, 2005; published online January 18, 2006.

Address correspondence and reprint requests to: Fon-Jou Hsieh, MD; E-mail: fjhsieh@ha.mc.ntu.edu.tw.

Published by Springer Science+Business Media, Inc. @ 2006 The Society of Surgical Oncology, Inc.
Neuroblastoma (NB) is an embryonic cancer of the postganglionic sympathetic nervous system that most commonly arises in the adrenal gland. It is one of the most common pediatric cancers, with an incidence of 
8.0 per million per year, ${ }^{1}$ and $96 \%$ of cases occur before the age of 10 years. ${ }^{2}$ Clinical and biological studies have discriminated NB into at least two distinct entities. ${ }^{3,4}$ One, favorable NB, is characterized genetically by near-triploid karyotypes with whole chromosome gains. These tumors are usually localized and frequently seen in patients $<1$ year of age or are detected by mass screening. The favorable NBs either regress spontaneously or differentiate into benign ganglioneuromas and hence lead to a good outcome with minimal or even no treatment. The other entity, unfavorable NB, is characterized by diploid karyotypes with structural changes, including deletion of $1 \mathrm{p}$ or $11 \mathrm{q}$, unbalanced gain of $17 \mathrm{q}$, and/or amplification of the myelocytomatosis viral-related oncogene, neuroblastoma-derived (MYCN) protooncogene. Patients with unfavorable NBs are usually older than 1 year of age and have advanced stages of disease and a very poor prognosis, even with aggressive treatment. These lines of evidence suggest that a more detailed understanding of the clinical and biological characteristics of NB may allow for a more appropriate intensity of therapy for these tumors. Indeed, recent progress in biological and genetic understanding of NB has led to risk-related therapy to improve therapeutic outcomes and avoid unnecessary treatments. ${ }^{5}$

For decades, there has been debate regarding the role of surgery in NB, especially in advanced NB. Surgical resection alone can provide adequate treatment for early-stage disease. ${ }^{6}$ However, the importance of tumor resection as a therapeutic maneuver in the treatment of advanced NB remains controversial. Some investigators have reported that gross total resection (GTR) of tumors improves the survival rate in advanced stages, ${ }^{7,8}$ whereas others have not found any improvement with GTR. ${ }^{9}{ }^{910}$ Most of these studies did not take into consideration the effect of biological factors of NB on the outcome of aggressive surgery, but considered only the clinical factors of disease stage and the patient's age. Biological factors, such as MYCN (a transcription factor) and Trk-A (a nerve growth factor receptor), have great influence on the tumor behavior of NB and the patient's outcome. ${ }^{11,12}$ NBs with MYCN amplification usually progress rapidly and have a very poor outcome, ${ }^{11}$ whereas tumors with high levels of Trk-A expression are susceptible to tumor differentiation or regression and have a favorable outcome. ${ }^{12}$ Neglecting these biological factors in the treatment of NB may misrepresent the true role of surgery in therapeutic strategies. In this study, we evaluated the effect of the biological factors MYCN and Trk-A on the outcome after GTR in advanced NB with the aim of determining the role of GTR in different biological groups of NBs.

\section{MATERIALS AND METHODS}

\section{Patients and Treatment}

From December 1990 to December 2002, 45 advanced-stage NB patients treated at National Taiwan University Hospital who had complete follow-up data were included in this study. Thirty-five patients had stage 4 tumors, and the other 10 had stage 3 tumors according to the International NB Staging System. ${ }^{13}$ Age, sex, primary tumor site, Shimada classification of pathology,${ }^{14}$ surgical complications, and the patient's outcome were also recorded.

All patients underwent an initial diagnostic biopsy. Surgical specimens were submitted to pathologic assessment as well as to analysis of molecular markers, including Trk-A expression and MYCN status, before chemotherapy. After biopsy, all patients received induction chemotherapy that consisted of cisplatin 60 $\mathrm{mg} / \mathrm{m}^{2}$ day $1, \mathrm{VP}-16100 \mathrm{mg} / \mathrm{m}^{2}$ and epirubicin $30 \mathrm{mg} /$ $\mathrm{m}^{2}$ day 2 , cyclophosphamide $900 \mathrm{mg} / \mathrm{m}^{2}$ days 3 and 4 , and VP-16 $60 \mathrm{mg} / \mathrm{m}^{2}$ day 5 . Each cycle was repeated every 4 weeks. GTR of the tumor was attempted after three to six cycles of chemotherapy. GTR was defined as removal of the macroscopic primary tumor and all regional lymph nodes, according to the operation record. There should be no grossly visible tumors detected by the immediate follow-up magnetic resonance imaging or computed tomographic scan studies 10 days after the definitive operation. A second-look operation might be needed to achieve GTR after initial partial resection and a subsequent additional chemotherapy response. Patients who underwent lesser procedures (partial excision and biopsy) were grouped as incomplete resection (IR) for comparison with the GTR group. After the definitive surgical procedure, patients received three to five cycles of maintenance chemotherapy (repeated induction regimen) or autologous bone marrow transplantation. The median follow-up after the diagnosis was 30 months (range, 5-144 months).

\section{Assessment of Trk-A Expression by Immunohistochemistry}

The details of immunostaining for the Trk-A gene product have been described previously. ${ }^{15}$ Trk-A expression was examined on sections from formalinfixed, paraffin-embedded tissue by an avidin-biotin complex immunoperoxidase staining technique. A 
rabbit anti-human Trk-A antibody (Upstate Biotechnology Inc., Lake Placid, NY) at a dilution of 1/ 150 was used as a primary antibody. Diaminobenzidine was used for visualization, and nuclei were counterstained with hematoxylin. One ganglioneuroma tumor with consistent Trk-A expression by immunohistochemistry was used as a positive control. Nonimmunized rabbit serum was used as a negative control. The immunoreactivity of Trk-A was recorded as follows: "negative" indicated that staining was absent throughout the specimen, and "positive" indicated that brownish granular staining was present in the cytoplasm of the NB or ganglion cells.

\section{Analysis of MYCN Amplification by Fluorescence In Situ Hybridization}

The MYCN status in NB tumors was evaluated by fluorescence in situ hybridization (FISH) analysis of formalin-fixed paraffin-embedded tissues or fresh single tumor cells. ${ }^{16,17}$ The nuclei on slides were denatured in $70 \%$ formamide $/ 2 \times$ standard saline citrate and rehydrated. The MYCN gene probe (spectrum orange) and the internal control TelVysion $2 p$ probe (spectrum green), acquired from Vysis (Downer's Grove, IL), were used for hybridization. Metaphase spreads were counterstained with $.5 \mathrm{mg} /$ $\mathrm{mL}$ of 4',6-diamidino-2-phenylindole in Vectashield (Vector, Burlingame, CA). Fluorescent signals representing the presence of the $\mathrm{MYCN}$ gene were counted for 100 cells on each slide. Cells with clusters or more than six grains of red fluorescence were considered evidence of MYCN gene amplification. ${ }^{17}$ Lymphocytes obtained from a healthy male donor were used as controls for the FISH study.

\section{Risk Grouping}

To further evaluate the influence of biological factors on the role of surgery in the prognosis of $\mathrm{NB}$, the prognosis of all patients was classified into three risk groups according to the two biological markers of TrkA and MYCN: low risk (positive Trk-A expression and normal MYCN copy number), intermediate risk (negative Trk-A expression and normal MYCN copy number), and high risk (MYCN amplification with positive or negative Trk-A expression).

\section{Statistical Analysis}

The statistical analyses were performed with SPSS 10.0 software for Windows (SPSS Inc., Chicago, IL). Associations between pairs of categorical variables were assessed with Pearson's $\chi^{2}$ test. Survival prob- abilities in various subgroups were estimated with the Kaplan-Meier method. Survival of groups was compared by log-rank tests. All statistical tests were two sided, and a $P$ value of $\leq .05$ was considered to be statistically significant.

\section{RESULTS}

There were 27 men and 18 women. The median age at diagnosis was 3.5 years (range, $.5-11.5$ years); 4 were $\leq 1$ year of age, and 41 were older than 1 year. The pathology was favorable in 17 tumors and unfavorable in 28 according to Shimada's classification. In 26 patients, the primary tumors were located in the adrenal gland; 9 were paraspinal, 5 mediastinal, 3 cervical, and 2 pelvic. After induction chemotherapy, GTR was achieved in 21 patients. Among 24 patients with incomplete tumor excision, 5 patients did not receive further tumor excision except for biopsy because they died from rapid tumor progression or chemotherapy complications. In the GTR group, there was no surgical mortality, but there was 1 chylothorax and there were 2 cases of adhesion ileus and 4 nephrectomies, for a total complication rate of $33 \%$ (7 of 21). The nephrectomies were necessitated by direct involvement of tumor with the renal parenchyma or vessels. In the IR group, there was 1 mortality (respiratory failure after biopsy of the mediastinal tumor) and 1 nephrectomy, for a total complication rate of $8 \%$ ( 2 of 24). The complication rate was significantly higher in the GTR group $(P=.036)$.

Fifteen $(33.3 \%)$ tumors had a variable percentage (12\%-93\%) of cells with positive signals of fluorescence of MYCN in FISH analysis and were recognized as having MYCN amplification (Fig. 1A and B). Seventeen $(37.8 \%)$ tumors had positive Trk-A immunostaining on NB or ganglion cells (Fig. 1C and D).

Further analysis of the association between GTR and various clinicopathologic and biological factors showed that GTR was significantly easier to achieve in tumors with low biological risk than in those with intermediate or high risk (12 of 13, 4 of 17, and 5 of 15 , respectively; $P<.001$; Table 1$)$. In addition, GTR also tended to be achieved in tumors with favorable histological characteristics $(P=.059)$.

Univariate analysis showed that GTR, favorable Shimada's histology, and positive Trk-A expression strongly correlated with better survival, whereas MYCN amplification predicted a very poor outcome (Table 2). In addition, the prognosis of all patients could be clearly discriminated by the biological risk grouping: (1) low risk (13 patients), predicted 5-year survival rate of $65.6 \%$; (2) intermediate risk (17 pa- 

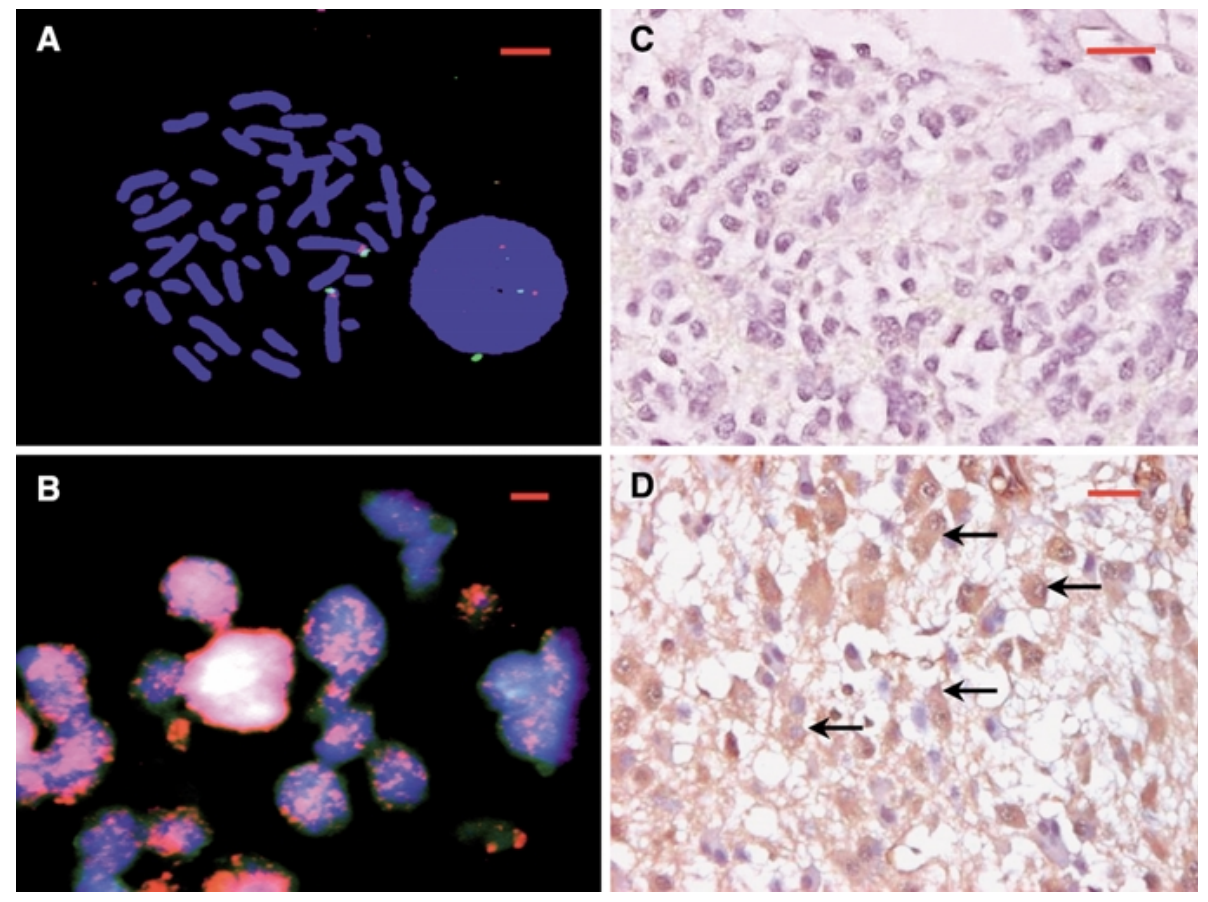

FIG. 1. Analysis of myelocytomatosis viral-related oncogene, neuroblastoma-derived (MYCN) status by fluorescence in situ hybridization (A and B) and immunohistochemical staining of Trk-A in neuroblastoma (C and D). (A) A normal control lymphocyte shows only 2 grains of red fluorescence in either interphase or metaphase. (B) A typical neuroblastoma tumor with MYCN amplification shows clusters and grains of red fluorescence in the blue nuclei. (C) Negative control for Trk-A immunostaining. (D) Positive Trk-A staining in neuroblastoma cells (arrows). Scale bars $=10 \mu \mathrm{m}$ for $(\mathbf{A})$ and (B); $100 \mu \mathrm{m}$ for (C) and (D).
TABLE 1. Association between GTR and clinicopathologic and biologic factors

\begin{tabular}{lrrr}
\hline Variable & $\mathrm{n}$ & GTR $(\%)$ & $P$ value \\
\hline Age at diagnosis (y) & & & \\
$\quad \leq 1$ & 4 & $1(25.0)$ & .363 \\
$\quad \quad 1$ & 41 & $20(48.8)$ & \\
Clinical stage & & & \\
$\quad 3$ & 10 & $5(50.0)$ & .811 \\
$\quad 4$ & 35 & $16(45.7)$ & \\
Primary tumor site & & & \\
$\quad$ Adrenal & 26 & $11(42.3)$ & .493 \\
$\quad$ Extra-adrenal & 19 & $10(52.6)$ & \\
Shimada histology & & & \\
$\quad$ Favorable & 17 & $11(64.7)$ & .059 \\
$\quad$ Unfavorable & 28 & $10(35.7)$ & \\
Risk group & & & \\
$\quad$ Low & 13 & $12(92.3)$ & $<.001$ \\
$\quad$ Intermediate & 17 & $4(23.5)$ & \\
$\quad$ High & 15 & $5(33.3)$ & \\
\hline
\end{tabular}

GTR, gross total resection.

tients), predicted 5-year survival rate of $32.1 \%$; and (3) high risk (15 patients), predicted 5-year survival rate of $0 \%$ (Fig. $2 ; P<.001$; log-rank test). Significant prognostic factors revealed by univariate analysis were further assessed by the multivariate Cox proportional hazards model, which showed that only the biological risk grouping was an independent prognostic factor (Table 3).

To further study the effect of biological risk grouping on the prognostic discrimination by surgical procedures, the survival of NB patients was further
TABLE 2. Univariate analysis of survival of 45 neuroblastoma patients

\begin{tabular}{llll}
\hline Variable & $\begin{array}{l}\text { Predicted } \\
\text { 5-y survival (\%) }\end{array}$ & $\begin{array}{l}\text { Hazard } \\
\text { ratio }\end{array}$ & $P$ value \\
\hline Age (y) & & & \\
$\quad \leq 1$ & 25.0 & 1.165 & .803 \\
$\quad>1$ & 32.66 & 1 & \\
Stage & & & .049 \\
$\quad 3$ & 71.4 & 1 & \\
$\quad 4$ & 23.1 & 2.875 & .208 \\
Primary tumor site & & & \\
$\quad$ Adrenal & 20.7 & 1.595 & \\
$\quad$ Extra-adrenal & 45.3 & 1 & \\
Shimada histology & & & \\
$\quad$ Favorable & 66.9 & 1 & .019 \\
$\quad$ Unfavorable & 36.3 & 2.456 & \\
MYCN & & & \\
$\quad$ Amplified & .0 & 3.452 & $<.018$ \\
$\quad$ Not amplified & 46.8 & 1 & \\
Trk-A expression & & & \\
$\quad$ Positive & 49.2 & 1 & \\
$\quad$ Negative & 21.1 & 2.377 & \\
Operation & & & \\
$\quad$ GTR & 52.3 & 1 & \\
$\quad$ IR & 16.7 & 3.394 & \\
\hline
\end{tabular}

MYCN, myelocytomatosis viral-related oncogene, neuroblastoma-derived; GTR, gross total resection; IR, incomplete resection.

stratified according to the risk grouping and surgical procedures (Fig. 3). For each risk group, GTR could provide prognostic value only for the intermediaterisk patients (Fig. 4; $P=.037$ ), but not for the lowrisk or high-risk patients. In 17 patients in the intermediate-risk group, 4 received GTR of their tumors, 


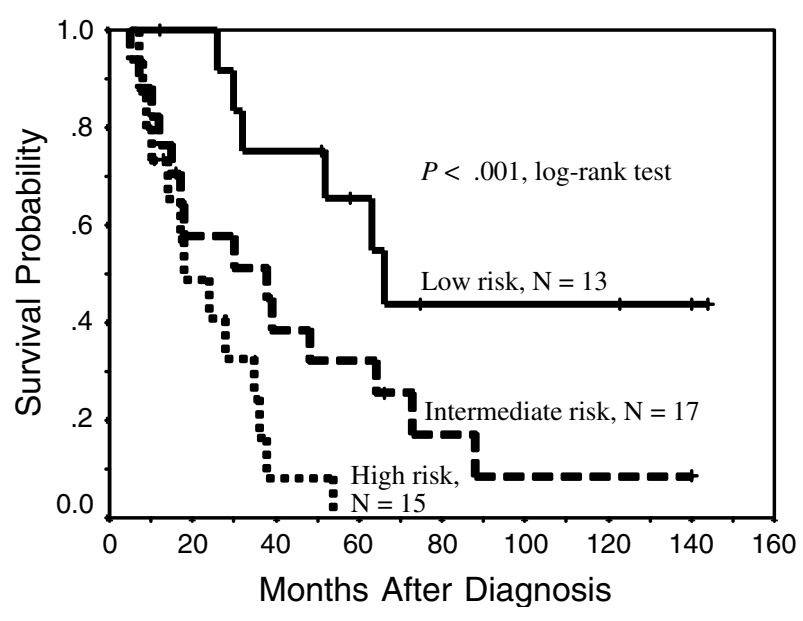

FIG. 2. Cumulative survival curves according to the biological risk grouping by Trk-A expression and myelocytomatosis viral-related oncogene, neuroblastoma-derived (MYCN) status of all 45 neuroblastoma patients.

TABLE 3. Multivariate analysis of survival of 45 neuroblastoma patients

\begin{tabular}{lccc}
\hline Variable & Relative risk & $95 \%$ CI & $P$ value \\
\hline Stage & 2.862 & $.986-8.309$ & .053 \\
Shimada histology & 1.325 & $.599-2.928$ & .487 \\
Operation $_{\text {Risk grouping }}{ }^{\mathrm{a}}$ & 2.215 & $.958-5.121$ & .063 \\
\hline
\end{tabular}

CI, confidence interval.

${ }^{\text {a }}$ Risk grouping by Trk-A expression and myelocytomatosis viralrelated oncogene, neuroblastoma-derived (MYCN) status.

with a predicted 5-year survival rate of $66.7 \%$, and the other 13 had IR of their tumors, with a predicted 5 -year survival rate of $23.1 \%$. All 13 patients of lowrisk group survived, and all except 1 received GTR. Five patients received GTR and 10 patients received IR in the high-risk group. All 15 patients in the highrisk group died within 5 years except for 2 patients with GTR of their primary tumors; these patients had bone and brain metastasis at 11 and 13 months' follow-up, respectively. From another point of view, of 24 patients who received IR of their tumors, only 1 patient belonged to the low-risk group and survived, and the prognosis of the 13 patients of the intermediate-risk group was as poor as that of the 10 patients in the high-risk group (the predicted 5-year survival rate was $23.1 \%$ and $0 \%$, respectively; Fig. $5 \mathrm{~A} ; P=$ .214). In 21 patients who received GTR of their tumors, the prognosis of the 4 patients in the intermediate-risk group was as favorable as that of the 12 patients from the low-risk group (the predicted 5-year survival rate was $66.7 \%$ and $62.3 \%$, respectively) and was significantly more favorable than that of the 5

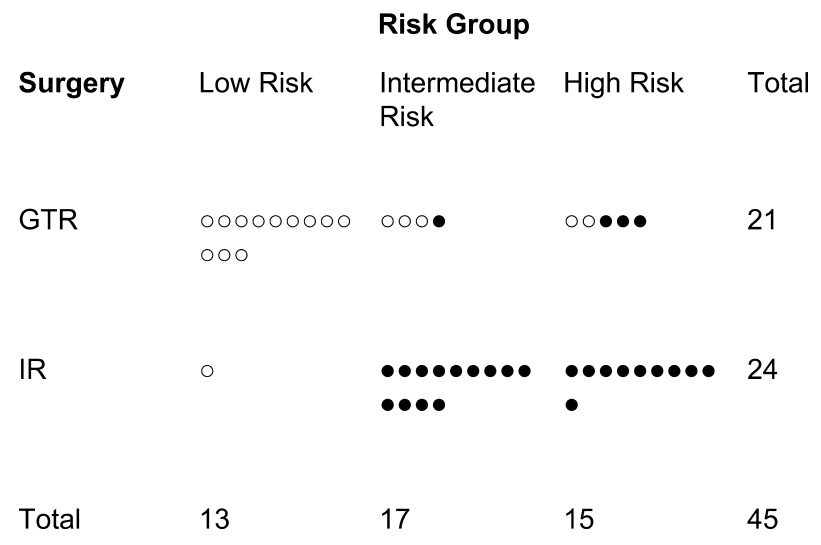

FIG. 3. Prognostic categories of biological risk grouping by Trk-A expression, myelocytomatosis viral-related oncogene, neuroblastoma-derived (MYCN) status, and surgical procedure in 45 neuroblastoma patients. Open circles represent survivors; filled circles represent patients who died. GTR, gross total resection; IR, incomplete resection.

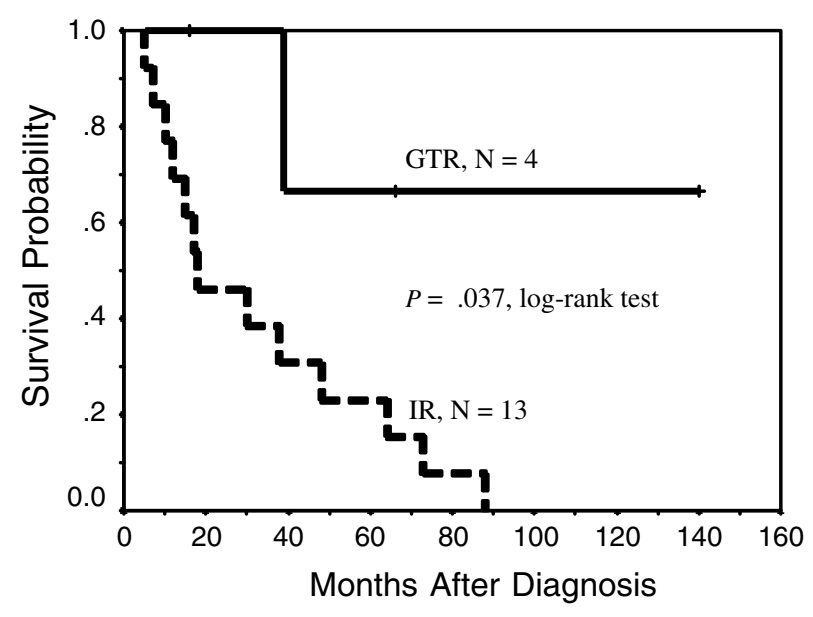

FIG. 4. Cumulative survival curves according to operative procedures in patients of intermediate biologic risk (negative Trk-A expression and normal myelocytomatosis viral-related oncogene, neuroblastoma-derived [MYCN] copy number). GTR, gross total resection; IR, incomplete resection.

patients in the high-risk group (the predicted 5-year survival rate was $0 \%$; Fig. $5 \mathrm{~B} ; P=.004$ ).

\section{DISCUSSION}

GTR of advanced NB is a high-risk procedure and is very challenging to pediatric surgeons, because the procedure usually requires dissection of the tumor from great vessels and other vital organs such as the kidneys, liver, and spinal cord. The surgical complication rate may be as high as $30 \%$, including $20 \%$ of 
A NB patients of IR group
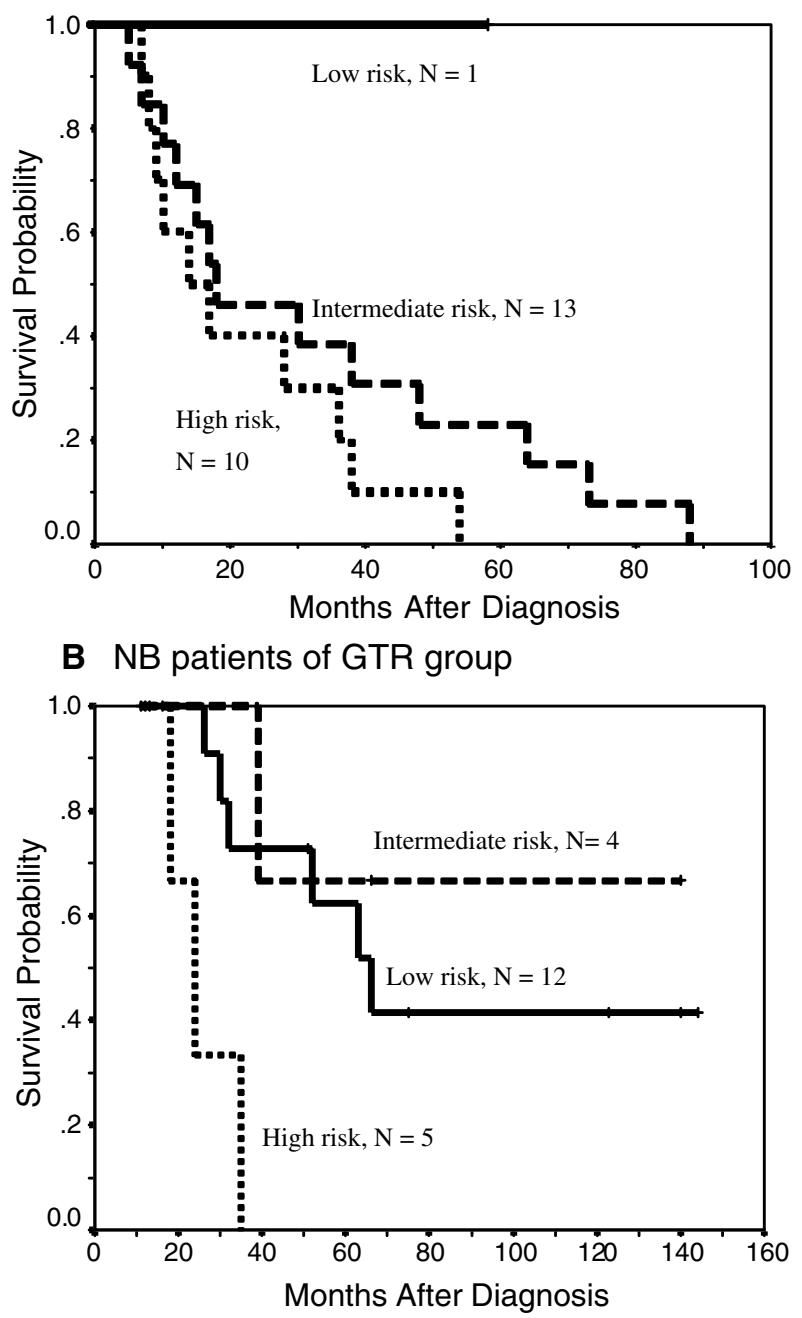

FIG. 5. Cumulative survival curves according to the biological risk grouping by Trk-A expression and myelocytomatosis viral-related oncogene, neuroblastoma-derived (MYCN) status in (A) neuroblastoma (NB) patients who had incomplete resection (IR) of their tumors and (B) NB patients who had gross total resection (GTR) of their tumors.

patients who have their healthy organs removed (in particular, their kidneys). ${ }^{18,19}$ However, it is not clear whether such a risky radical resection can achieve a long-term remission for advanced NBs. Several reports have shown that although GTRs contribute to the control of local recurrence, the overall survival of NB patients depends largely on the resolution of distant metastasis. ${ }^{8,10,20}$ One important reason for such a dilemma is probably the marked biological heterogeneity of NB. More than 195 tumor markers of NB have been studied. ${ }^{21}$ The biological heterogeneity may result in diverse responses to a single surgical strategy. Therefore, further biological classification to guide the surgical treatment of advanced NB would be very helpful.

This study was designed to use two important biological markers, MYCN and Trk-A, by which the patients were classified into three risk groups, to define the effect of biological makers on the role of surgery in NB. We showed that GTR could provide prognostic value only for NB patients in the intermediate biological risk group (negative Trk-A expression and normal MYCN copy number). However, GTR did not provide prognostic value for NB patients with high biological risk (MYCN amplification with positive or negative Trk-A expression) as a result of the extremely poor prognosis of these patients. Further intensified chemotherapy regimens are required to improve their survival. ${ }^{22,23}$

NB patients at low biological risk (positive Trk-A expression and normal MYCN copy number) had an overall favorable outcome (all 13 patients survived). In addition, because low biological risk NBs tend to be more localized, ${ }^{24}$ these tumors should be easier to totally resect (12 of 13 patients received GTR). Therefore, it is difficult to analyze the true effect of different surgical procedures on the prognosis of these patients. We do not know whether the favorable outcome of these low-risk patients is due to their favorable biological properties or to GTR. Although some studies have shown that low biological risk patients with IR of their tumors would still have a favorable outcome, ${ }^{24,25}$ it is ethically inappropriate to perform IR in these patients for a controlled randomized study. Nevertheless, our multivariate analysis showed that biological risk grouping was the only single independent prognostic factor, thus suggesting that biological prognostic factors have a great effect on patient survival regardless of the surgical method.

However, our analysis further showed that in the IR group, patients with intermediate biological risk had a poor prognosis similar to that of the high-risk patients, whereas in the GTR group the prognosis of the intermediate-risk patients was as favorable as that of the low-risk patients. This result indicates that in patients with selective biological risks, surgical procedures do alter the disease's natural outcomes.

It has been shown that GTR or partial resection of advanced NB with MYCN amplification carries similar outcomes, but both procedures did carry a better survival than simple biopsy. ${ }^{25,26}$ This may be due to the very poor biological nature of some NBs with MYCN amplification, which progressed rapidly after a biopsy and never underwent delayed operation. In addition, only one third (15 of 45) of advanced NBs show MYCN amplification, and this 
suggests that advanced NB without MYCN amplification (predictive 5-year survival rate of $46.8 \%$ ) is a large, heterogeneous group. Therefore, additional biological markers, such as Trk-A, are needed to further discriminate these heterogeneous tumors.

Our result showed that GTR carried a higher complication rate than IR did. This difference was mainly because more nephrectomies were performed in the GTR group than the IR group (four and one, respectively). Nephrectomy may be needed to achieve GTR in selected patients whose tumors directly invade the kidney or the renal vessels. However, this result was not found in other larger group studies. ${ }^{8,19}$ There are two possible causes for this discrepancy: first, the small patient numbers in our study may bias the complication rate; second, in larger centers of $\mathrm{NB}$, the risk of nephrectomy may be reduced by more experience with surgery of advanced NB. ${ }^{8,19}$

GTR is a serious and often life-threatening operation. However, in this study of a small patient population, GTR carried prognostic benefit in only a selected intermediate-risk biological group of NB patients. GTR did not show a beneficial effect in NB patients with high biological risk tumors because of the overall poor prognosis of these patients. For NB patients of low biological risk, it was difficult to analyze the effect of different surgical procedures on their prognosis because of the overall favorable outcome and the high GTR rate in these patients. Further studies with large patient populations are needed to clarify the influence of biological factors on surgical strategies for advanced NB.

\section{ACKNOWLEDGMENTS}

Supported by a grant from the National Taiwan University Hospital (NTUH 92-M016), Taipei, Taiwan.

\section{REFERENCES}

1. Gurney JG, Severson RK, Davis S, Robison LL. Incidence of cancer in children in the United States: sex-, race- and 1-year age-specific rates by histologic type. Cancer 1995; 75:2186-95.

2. Grovas A, Fremgen A, Rauck A, et al. The National Cancer Data Base report on patterns of childhood cancers in the United States. Cancer 1997; 80:2321-2.

3. Woods WG, Lemieux B, Tuchman M. Neuroblastoma represents distinct clinical-biologic entities: a review and perspective from the Quebec Neuroblastoma Screening Project. Pediatrics 1992; 89:114-8.

4. Brodeur GM. Neuroblastoma: biologic insights into a clinical enigma. Nat Rev Cancer 2003; 3:203-16.

5. Castleberry RP. Pediatric update: neuroblastoma. Eur $J$ Cancer 1997; 33:1430-8.

6. Matthay KK, Sather HN, Seeger RC, Haase GM, Hammond GD. Excellent outcome of stage II neuroblastoma is indepen- dent of residual disease and radiation therapy. $J$ Clin Oncol 1989; 7:236-44.

7. Haase GM, O'Leary MC, Ramsay N, et al. Aggressive surgery combined with intensive chemotherapy improves survival in poor-risk neuroblastoma. J Pediatr Surg 1991; 26:1119-24.

8. La Quaglia MP, Kushner BH, Heller G, et al. The impact of gross total resection on local control and survival in high-risk neuroblastoma. J Pediatr Surg 2004; 39:412-7.

9. Kaneko M, Ohakawa H, Iwakawa M. Is extensive surgery required for the treatment of advanced neuroblastoma? $J$ Pediatr Surg 1997; 32:1616-9.

10. Castel V, Tovar JA, Costa E, et al. The role of surgery in stage IV neuroblastoma. J Pediatr Surg 2002; 37:1574-8.

11. Seeger RC, Brodeur GM, Sather H, et al. Association of multiple copies of the $\mathrm{N}$-myc oncogene with rapid progression of neuroblastomas. $N$ Engl J Med 1985; 313:1111-6.

12. Nakagawara A, Arima-Nakagawara M, Scavarda NJ, Azar CG, Cantor AB, Brodeur GM. Association between high levels of expression of the TRK gene and favorable outcome in human neuroblastoma. $N$ Engl J Med 1993; 328:847-54.

13. Brodeur GM, Prichard J, Berthold F, et al. Revision of the international criteria for neuroblastoma diagnosis, staging, and response to treatment. J Clin Oncol 1993; 11:1466-77.

14. Shimada H, Chatten J, Newton WA, et al. Histopathologic prognostic factors in neuroblastic tumors: definition of subtypes of ganglioneuroblastoma and an age-linked classification of neuroblastomas. J Natl Cancer Inst 1984; 73:405-16.

15. Tanaka T, Hiyama E, Sugimoto T, Sawada T, Tanabe M, Ida N. trk A gene expression in neuroblastoma: the clinical significance of immunohistochemical study. Cancer 1995; 76:1086-95.

16. Sartelet H, Grossi L, Pasquier D, et al. Detection of N-myc amplification by FISH in immature areas of fixed neuroblastomas: more efficient than Southern blot/PCR. J Pathol 2002; 198: 83-91.

17. Grady-Leopardi EF, Schwab M, Ablin AR, Rosenau W. Detection of N-myc oncogene expression in human neuroblastoma by in situ hybridization and blot analysis: relationship to clinical outcome. Cancer Res 1986; 46:3196-9.

18. Adkins ES, Sawin R, Gerbing RB, London WB, Matthay KK, Haase GM. Efficacy of complete resection for high-risk neuroblastoma: a Children's Cancer Group study. J Pediatr Surg 2004; 39:931-6.

19. Shamberger RC, Smith EI, Joshi VV, et al. The risk of nephrectomy during local control in abdominal neuroblastoma. J Pediatr Surg 1998; 33:161-4.

20. Matsumura M, Atkinson JB, Hays DM, et al. An evaluation of the role of surgery in metastatic neuroblastoma. J Pediatr Surg $1988 ; 23: 448-53$.

21. Riley RD, Heney D, Jones DR, et al. A systemic review of molecular and biologic tumor markers in neuroblastoma. Clin Cancer Res 2004; 10:4-12.

22. Garaventa A, Boni L, Lo Piccolo GP, et al. Localized unresectable neuroblastoma: results of treatment based on clinical prognostic factors. Ann Oncol 2002; 13:956-64.

23. Kaneko M, Tsuchida $\mathrm{Y}$, Mugishima $\mathrm{H}$, et al. Intensified chemotherapy increases the survival rates in patients with stage 4 neuroblastoma with MYCN amplification. J Pediatr Hematol Oncol 2002; 24:613-21.

24. Cheung NKV, Kushner BH, LaQuaglia MP, et al. Survival from non-stage 4 neuroblastoma without cytotoxic therapy: an analysis of clinical and biologic markers. Eur J Cancer 1997; 33:2117-20.

25. von Schweinitz D, Hero B, Berthold F. The impact of surgical radicality on outcome in childhood neuroblastoma. Eur $J$ Pediatr Surg 2002; 12:402-9.

26. Nakagawara A, Ikeda K, Yokoyama T, Tsuda T, Higashi K. Surgical aspects of N-myc oncogene amplification of neuroblastoma. Surgery 1988; 104:34-40. 